\title{
ANÁLISIS DE LOS EFECTOS ELECTROMAGNÉTICOS DE LA INTEGRACIÓN DE UN ARREGLO DE ANTENA DE 4 ELEMENTOS CON LA ESTRUCTURA DE UN TELÉFONO PARA BANDAS MILIMÉTRICAS.
}

\author{
${ }^{1}$ Mark Clemente Arenas ${ }^{\text {dol }},{ }^{1}$ Abimael Erasmo Huamani Mallma, ${ }^{1}$ Brayan David \\ Wong Avila \\ ${ }^{1}$ Universidad Nacional Tecnológica de Lima Sur, Lima, Perú
}

Recibido: 05/09/2021 Revisado: 03/10/2021 Aceptado: 16/11/2021 Publicado: 30/01/2022

\section{Resumen}

Este artículo presenta un diseño de un arreglo de 4x1 antenas con alimentación microcinta coplanar gruesa de $100 \mathrm{Ohms}$ que opera alrededor de los 24, 26 y $28 \mathrm{GHz}$ para bandas de comunicaciones de quinta generación 5G. Los elementos que componen el arreglo son antenas de parche y las capas de cobre que utilizan la técnica de apertura de acoplamiento de multicapas para mejorar el rendimiento en lo referente a la ganancia y directividad. Utilizando herramientas de simulación se obtienen las pérdidas de retorno, el patrón de radiación, intensidad de campo y ganancia realizada. Finalmente se presenta una evaluación de los efectos en la propagación de las estructuras de teléfonos móviles actuales y de las cubiertas de protección en el campo cercano reactivo y radiante.

\begin{abstract}
This paper presents a design of a 4x1 antenna array with 100 Ohms thick coplanar microstrip feed operating around 24, 26 and $28 \mathrm{GHz}$ for fifth generation 5G communications bands. The elements composing the array are patch antennas and the copper layers that use the multilayer coupling aperture technique to improve performance in terms of gain and directivity. Using simulation tools, return loss, radiation pattern, Efield distribution and realized gain are obtained. Finally, an evaluation of the propagation effects of current cell phone structures and protective covers on the reactive and radiative near field is presented.
\end{abstract}

\section{Keywords}

Apertura de acoplamiento, arreglo de antenas, frecuencias milimétricas.

\section{Introduccion}


La quinta generacion (5G) y la posterior evolución B5G de redes móviles significan una gran revolucion global para las telecomunicaciones (Roh et al, 2014), (Marcus, 2015). Esta evolución tecnológica ha introducido el uso de nuevos segmentos del espectro radioeléctrico dentro del rango de los $25 \mathrm{GHz}$ a $76 \mathrm{GHz}$. Este rango de frecuencia es comúnmente conocido como bandas milimétricas o $\mathrm{mmW}$. Mas de tres rangos de frecuencia han sido identificado y ya se han ido desplegando en muchos lugares del mundo. Los segmentos de alrededor de $28 \mathrm{GHz}, 36 \mathrm{GHz}, 60 \mathrm{GHz}$ y $72 \mathrm{GHz}$ son los que corresponden a las bandas mmW de 5G (Elkashlan et al, 2015), ITU, 2020) o 5G mmW. En el caso de B5G, se utilizaran bandas similares o superiores a las mencionadas (Samsung, 2020), (Niu et al, 2015). El interés en el uso de las bandas mmW se debe a la enorme disponibilidad de ancho de banda disponible para las comunicaciones inalámbricas (Rappaport et al, 2013). Sin embargo, esta ventaja trae limitaciones relacionadas al alcance del enlace inalámbrico, ya que las perdidas de espacio libre se hacen demasiado considerables (Farooq \& Rather,2019). Para compensar esta desventaja se usan arreglos de antena para multiplicar la ganancia de las antenas y así compensar las perdidas de trayecto (Lin \& Ziolkowski, 2020). Es así que, se presenta el diseño de un arreglo de 4x1 antenas que opera en el rango $26 \mathrm{GHz}$ a $28 \mathrm{GHz}$ para teléfonos con modem 5G. Algunos trabajos disponibles en la literatura se han documentado la problemática de la performance de estos dispositivos cuando obstáculos, antes insignificantes, como el dedo humano (Nguyen et al, 2020) y o la piel humana (Lak et al 2021), (Sacco et al, 2021) se interponen en distancias cortas dentro de su campo cercano reactivo y radiante. Es así, que el presente articulo, tiene por finalidad realizar una evaluación de los efectos de cubiertas típicamente usadas para proteger teléfonos móviles en los parámetros circuitales y espaciales de la antena. Se uso un software de simulación electromagnética para analizar las perdidas de retorno, patrón de radiacion, Intensidad de campo eléctrico y ganancia realizada. Una discusión se presenta al final para subrayar los efectos encontrados.

\section{Método de evaluación}

\section{Diseño del arreglo de antenas milimétricas}

El diseño de esta antena está basado en el modelo de apilamiento de capas de circuito impreso o PCB stack- up. En bandas mmW, las perdidas producidas para transportar la energía RF desde los módems hasta las antenas en los teléfonos inalámbricos llega a valores importantes(Pan et al, 2012). Es así, que se usan diseños de antena que usen la 
superficie de empaquetado de los módems, como los que se presentan en (Lin \& Ziolkowski, 2020), (Sun, 2018), (Zhang \& Liu, 2009). Estos diseños usan diferentes las capas de cobre apiladas una encima de otra empleando técnica de acoplamiento por apertura. El circuito impreso empaquetado esta está constituido por 4 capas de cobre y tres capas de substrato los cuales tienes unas dimensiones de $\mathrm{Lg}=18 \mathrm{~mm}$ y Wg=39mm. En la capa superior se utilizan patch flotantes que permiten direccionar la propagación de las ondas en la dirección perpedicular, como se puede ver en la Figura 1.a. En la parte inferior el arreglo de antenas se alimenta mediante una línea de transmisión microstrip coplanar, como se ve en la Figura 1.b. En la parte superior, como se ve en la Figura 1.c, se observa los 4 patchs alimentados mediante acoplamiento a través de la capa superior metálica que cubre al tercer substrato de RO4350. La vista de perfil de la estructura final de la antena se compone de diferentes capas que se muestran en la Figura 1.c. Entre la capa inferior, se encuentra el primer substrato RO4350 $\left(\varepsilon_{r}=3.66, \tan \delta_{d}=0.001\right)$; Para el segundo substrato se usa Prepeg $\left(\varepsilon_{r}=3.96, \tan \delta_{d}=0.001\right)$ y en el tercero se vuelve a usar una lámina de RO4350. Todos los substratos se encuentran separados entre si por capas de cobre, con espesor igual a la de los patches. Cada capa metálica presenta una apertura. Sobre el primer substrato la apertura tiene una área de 5.6x1 $\mathrm{mm}^{2}$. Sobre el segundo y tercer substrato, la apertura tiene un área de $5.4 \times 0.58 \mathrm{~mm}^{2}$. Los cuatro patches superiores están a una distancia de $\mathrm{h}_{\mathrm{p}}=2.01 \mathrm{~mm}$ de las demás. En la Figura 1.d se ubica la antena en un modelo CAD de un teléfono móvil típico con banda 5G.

Por otro lado, todas las capas de cobre (amarillo) tienen un grosor de $18 \mu \mathrm{m}$. Los materiales de los substratos tienen una altura $h_{r o}=0.254 \mathrm{~mm}, h_{\text {pre }}=0.48 \mathrm{~mm}, h_{r o}=0.254$ $\mathrm{mm}$, respectivamente. La línea de transmisión en la capa inferior excita los slots de las capas superiores. Esta línea de transmisión está formada por 3 partes con las siguientes áreas $0.8 \times 1.5 \mathrm{~mm}^{2}-0.65 \times 1.5 \mathrm{~mm}^{2}-0.58 \times 6.5 \mathrm{~mm}^{2}$. El final de línea de transmisión esta encerrada dentro de una cavidad de apertura y se le rodea de vías para bloquear las ondas de superficie entre las 4 líneas de alimentación. Esto cumple la función de disminuir el acoplamiento entre elementos. El diámetro de estas vías es $0.25 \mathrm{~mm}$ y la distancia entre dos vías es $1.5 \mathrm{~mm}$.

Para alimentar las antenas es necesario calcular la impedancia de entrada característica de una línea complanar gruesa. Esta se puede calcular usando la ecuación ( 1 ). 


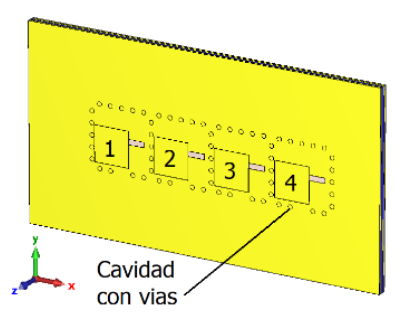

a)

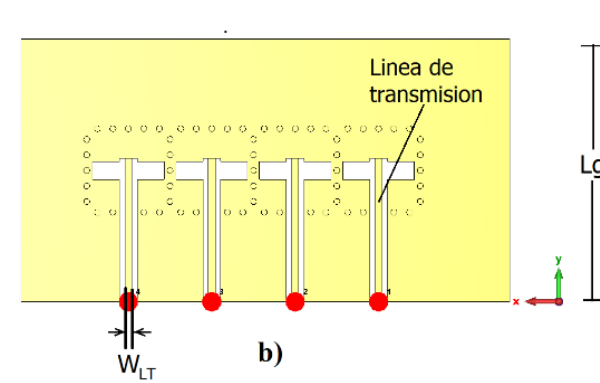

b)

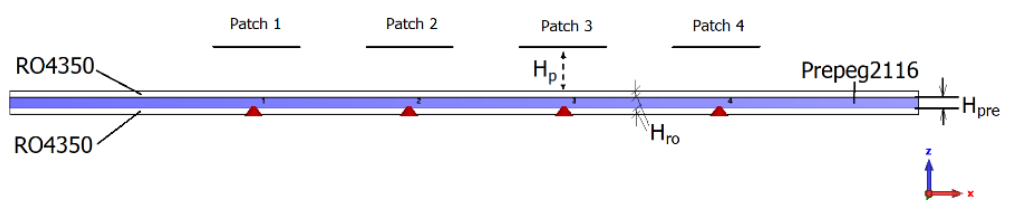

d)

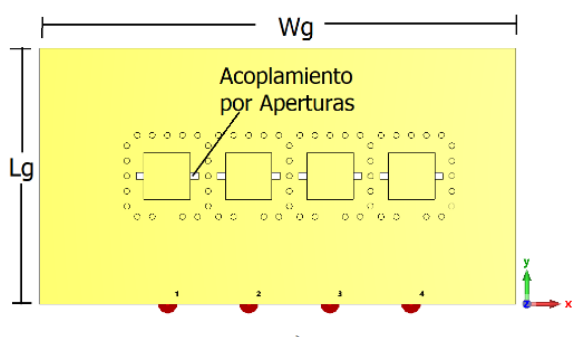

c)

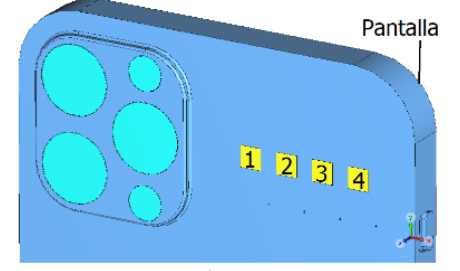

e)

Figura 1 Antena con arreglo de 4 elementos (a) Vista de perspectiva de la capa superior. b) Vista inferior de la capa 1. c) Vista superior de la antena. D) Vista de perfil de la antena. E) Vista de perspectiva de la antena dentro de teléfono.

$$
\begin{array}{r}
z=\frac{60 \pi}{\sqrt{\varepsilon_{r e}}} \frac{1}{\frac{K\left(k_{1}\right)}{K^{\prime}\left(k_{1}\right)}+\frac{K\left(k_{3}\right)}{K^{\prime}\left(k_{3}\right)}} \\
k_{1}=\frac{W_{T L}}{W_{T L}+2 * g a p}, \quad k_{3}=\frac{\tanh \left(\frac{\pi W_{T L}}{4 h_{r o}}\right)}{\tanh \left(\frac{\pi\left(W_{T L}+2 * g a p\right)}{4 h_{r o}}\right)}
\end{array}
$$

Donde: $\varepsilon_{r e}$, es la permitividad relativa del substrato. $K(k)$ y $K^{\prime}(k)$ representan la integral elíptica completa del tercer tipo y su complemento y se calculan mediante la ecuación ( 2 ) .

En el presente caso, la línea de alimentación coplanar tiene una impedancia de entrada de $100 \mathrm{Ohm}$, para un ancho de $\mathrm{W}_{\mathrm{TL}}=0.58 \mathrm{~mm}$ y una separación entre la línea y la metalización paralela de gap $=0.46 \mathrm{~mm}$ a cada lado. Considerando además que tiene una altura de metalización de $0.018 \mathrm{~mm}$ y $\mathrm{h}_{\mathrm{ro}}$ del primer sustrato.

En la parte superior se utilizan 4 patch de 4 x $4 \mathrm{~mm}^{2}$ con una separación de $3 \mathrm{~mm}$ entre ellos. Estos patchs están ubicados a $2 \mathrm{~mm}$ arriba de la capa superior con una separación de aire o $\varepsilon_{r}=1$. 


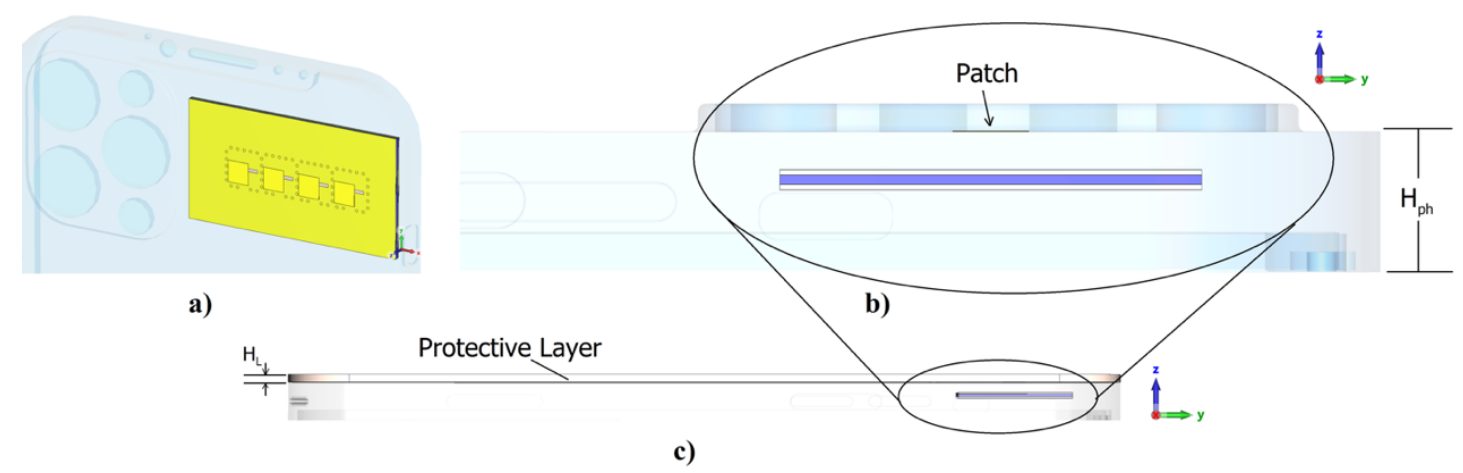

Figura 2 a) Arreglo de antenas de 4 elementos dentro del teléfono móvil. b) Vista de acercamiento del perfil del arreglo de antenas en el plano YZ. e) Vista lateral del teléfono móvil con la capa protectora.

\section{Resultados de Simulación y Discusiones}

La antena presentada en la Figura 1se posiciona en el teléfono móvil, como se muestra en la Figura 2.a y 2b. Se realizaran simulaciones considerando solo el plano de tierra producido por la pantalla del teléfono, el resto de componentes se omitirá para reducir el tiempo de la simulaciones, Considerando además que el efecto de tierra de la pantalla es la contribución mas notoria del teléfono a la antena. En seguida, se realizaran simulaciones considerando una capa protectora típica, ya que es frecuentemente utilizada como protección mecánica del teléfono, contra caídas o ralladuras. Los materiales usados para estas carcasas de protección varían desde plásticos, siliconas, cuero, madera, etc. Todos estos presentan diferentes constantes dieléctricas o permitividad relativa. Es importante tener en cuenta que mientras mas alta la permitividad será mayor el efecto en la propagación de campos a distancias cortas de la antena. Es por eso que se seleccionaron materiales con baja permitividad como el teflón $\left(\varepsilon_{r}=2.2, \tan \delta_{d}=0.0002\right)$, un policarbonato Poly $\left(\varepsilon_{r}=3, \sigma_{\text {cond }}=0.075\right)$, usado típicamente en carcasas, y el FR4 $\left(\varepsilon_{r}=4, \sigma_{\text {cond }}=0.025\right)$ con un espesor de $1.5 \mathrm{~mm}$. Todas las simulaciones se realizaron en el software comercial que resuelve las ecuaciones de Maxwell en el dominio del tiempo. Los resultados y el análisis de los efectos de la carcasa y el teléfono en el rendimiento de la antena se muestran a continuación. Para el presente análisis se consideraron los resultados de las pérdidas de retorno, patrón de radiación, densidad de campo eléctrico y ganancia realizada. 


\section{a). Análisis de las pérdidas de retorno}

Las perdidas de retorno indican la proporción de ondas reflejadas en comparación a las incidentes a la entrada de la antena. Es un parámetro útil que se relaciona a la obtención de ancho de banda de impedancia. Los cuatro puertos en las antenas están conectados a una impedancia de $100 \mathrm{Ohms}$. Las simulaciones dan como resultado los parámetros $\mathrm{S}$, de los cuales, la magnitud del parámetro $\left|\mathrm{S}_{11}\right|$ en $\mathrm{dB}$ es equivalente a las perdidas de retorno. Las perdidas de retorno de todos los puertos se presentan en la Fig. 3. Se presentan 5 casos: la antena de la Figura 1.a se usa como antena de referencia (curva roja); la antena de referencia dentro del modelo de teléfono móvil con plano de tierra (curva verde); la antena de referencia dentro del modelo de teléfono móvil con plano de tierra y una capa protectora de material FR4 (curva violeta); la antena de referencia dentro del modelo de teléfono móvil con plano de tierra y una capa protectora de material Policarbonato; finalmente, la antena de referencia dentro del modelo de teléfono móvil con plano de tierra y una capa protectora de material Teflon PTFE (curva azul.)
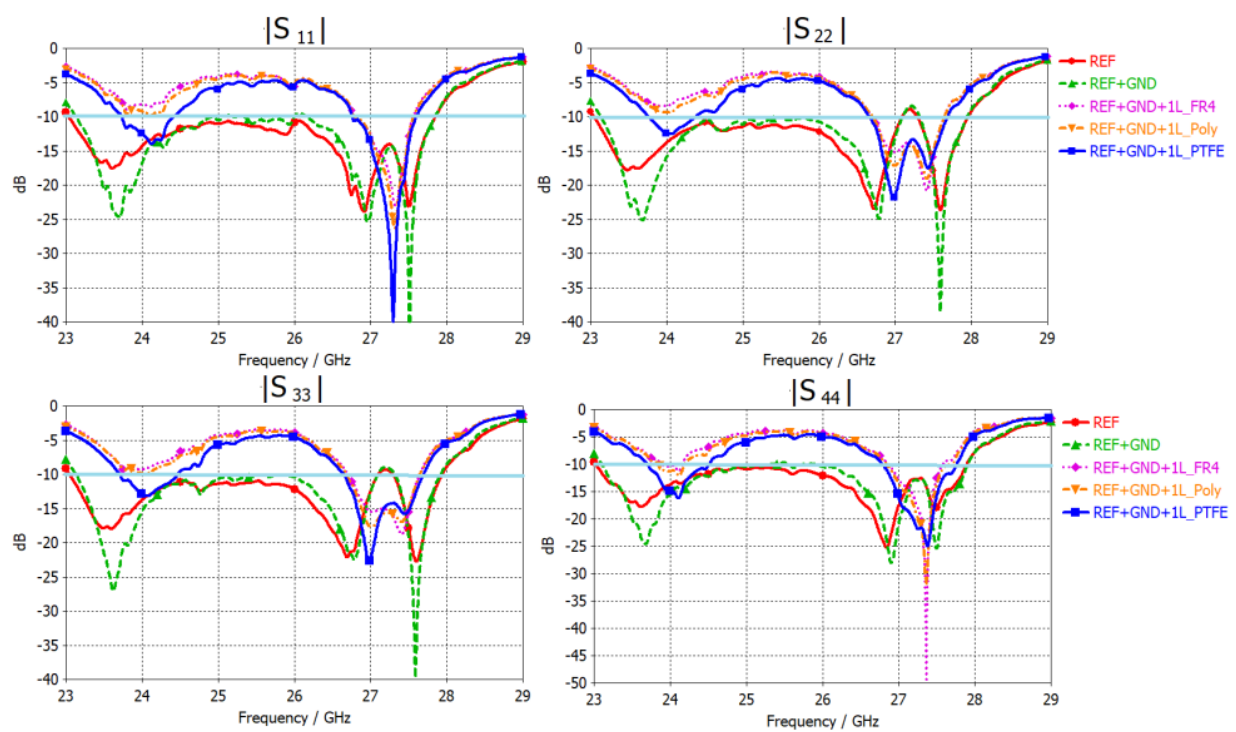

Figura 3 Pérdidas de retorno de la primera (1) a la cuarta (4) antena o elemento del arreglo.

La Figura 3 muestra que las pérdidas de retorno para la antena de referencia y con plano de tierra se mantienen por debajo de -10dB para frecuencias desde $23.08 \mathrm{GHz}$ hasta 27.95 $\mathrm{GHz}$, lo que indica un ancho de banda bastante grande de $\mathrm{BW}=4,87 \mathrm{GHz}$. Cuando se usan las capas protectoras de cualquier material, dicho ancho de banda se reduce a dos segmentos pequeños alrededor de $24 \mathrm{GHz}$ y $27.3 \mathrm{GHz}$. Los segmentos tienen un ancho de $670 \mathrm{MHz}$ alrededor de 24GHz. Esta banda existe solo en caso de la capa FR4, ya que para 
las otras capas no existe banda utilizable hasta los $26.7 \mathrm{GHz}$. Alrededor de $27.3 \mathrm{GHz}$ existen bandas utilizables de $820 \mathrm{MHz}$ para las tres capas protectoras. En otras palabras en términos de ancho de banda, el utilizar capas protectoras sobre la antena una reducción de hasta $4 \mathrm{GHz}$ o $83 \%$ del ancho de banda inicial. Una solución que se usa normalmente es cambiar el criterio de $-10 \mathrm{~dB}$ para el ancho de banda, con uno de $-6 \mathrm{~dB}$, lo que sirve para aumentar el ancho de banda hasta $2.97 \mathrm{GHz}$ alrededor de los dos nodos.

\section{b). Patrón de radiación}

El patrón de radiación es una representación que nos permite observar como parámetros como la directividad se comportan de manera espacial y son mostrados en la Figura 4. Los patrones de radiación en 3D se presentan en el mismo orden de la sección anterior, antena de referencia, antena de referencia con plano de tierra, antena de referencia con plano de tierra y capa de teflón, antena de referencia con plano de tierra y capa de policarbonato, antena de referencia con plano de tierra y capa de FR4.

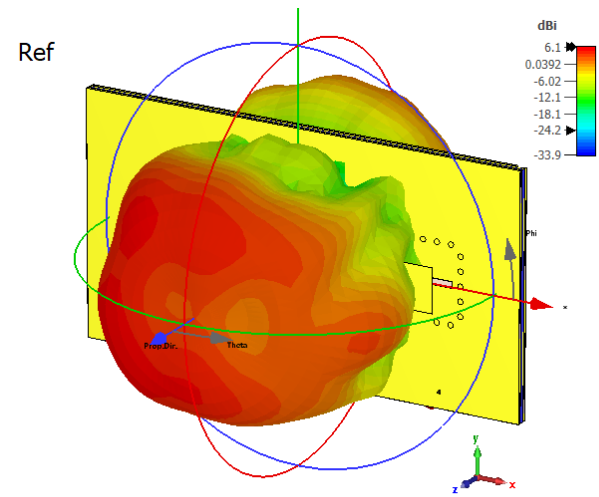

a)

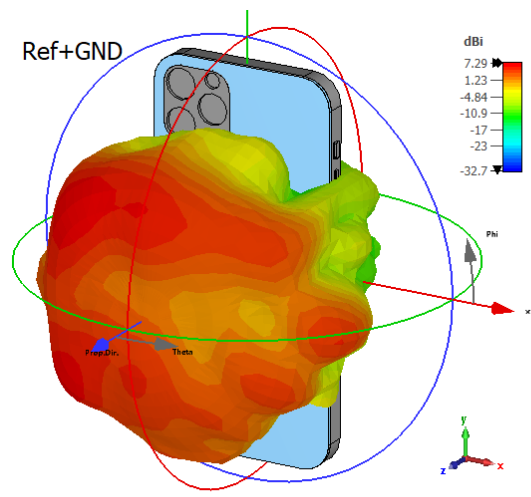

b)

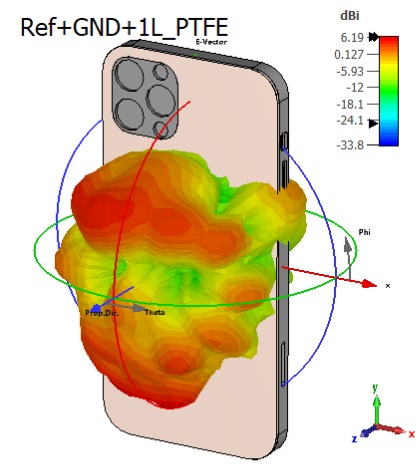

c)

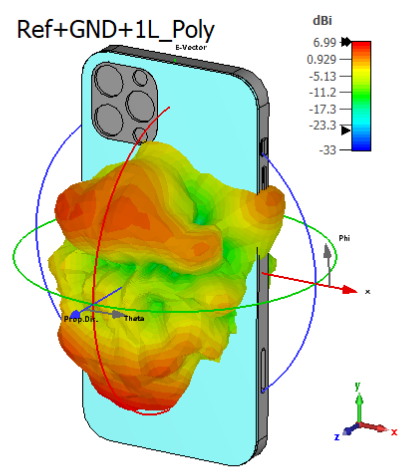

d)

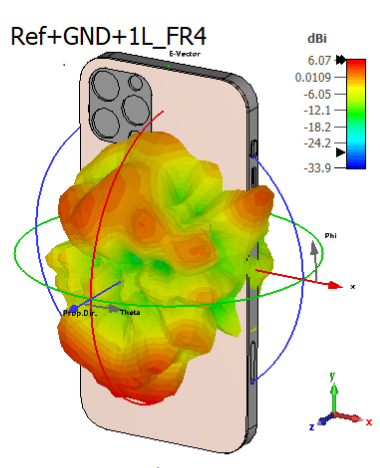

e)

Figura 4 Patrón de radiación de campo lejano. a) Antena acoplada por apertura de referencia. b) Antena de referencia dentro del teléfono mas plano de tierra. c) Antena de referencia dentro del teléfono mas plano de tierra mas capa protectora de Teflon, 
d)Antena de referencia dentro del teléfono mas plano de tierra mas capa de Poly, e) Antena de referencia dentro del teléfono mas plano de tierra mas capa de FR4

Los efectos son similares a los experimentados en el análisis del ancho de banda, ya que los casos de la antena referencia sola y con plano de tierra, el patrón de radiación se mantiene concentrado en la dirección máxima de propagación paralela al eje Z, como se ve en Figura 4.a y b. Es mas, en el caso de la antena con el plano de tierra, que actúa como reflector, la propagación se concentra en el eje $\mathrm{Z}$ y la ganancia máxima se incrementa de 6.1 a $7.27 \mathrm{~dB}$. Cuando se usa la capa protectora, esta produce nodos en la dirección máxima de propagación como se ve en las Figura 4.c, d y e. Los tres casos presentan nodos en el eje $\mathrm{Z}$, haciendo que el lóbulo principal se reoriente hacia $\theta=67^{\circ}$ y $\phi=47^{\circ}$ en el peor de los casos (FR4). En los tres casos, la máxima ganancia en cualquier dirección se ha reducido hasta valores cercanos al de la antena referencia: $6.19 \mathrm{~dB}$ (Teflon), $6.99 \mathrm{~dB}$ (Poly) y 6.07 (FR4). La capa protectora que presenta este efecto de manera mas reducida es la de Teflón.

\section{c). Campo Eléctrico Cercano}

Para poder entender lo que sucede cuando las ondas interactúan con la capa de protección al salir de la antena, es necesario observar la intensidad de campo eléctrico a distancias cercanas o campo cercano. La Figura 5, presenta la distribución espacial de la intensidad de campo eléctrico en el plano $\mathrm{YZ}$ cuando $\mathrm{x}=0$. La captura de la intensidad se realizo en todos los casos para una amplitud lineal de campo con una fase de $0^{\circ}$.

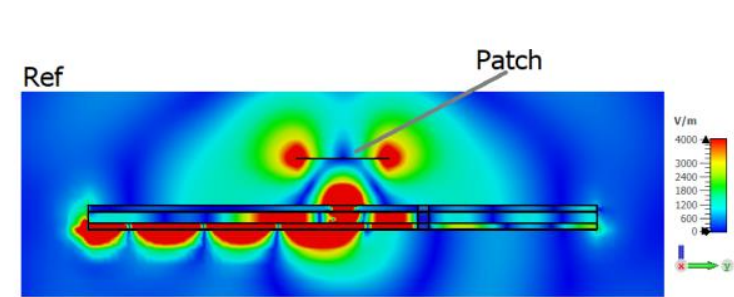

a)

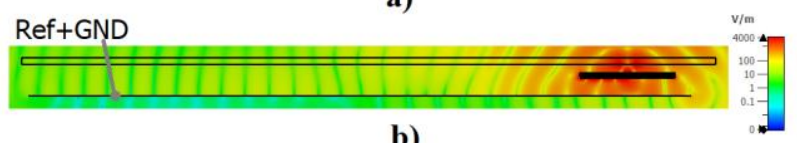

b)

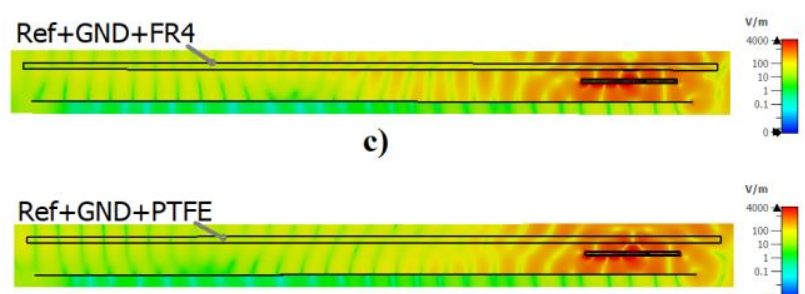

d)

Ref+GND+Poly

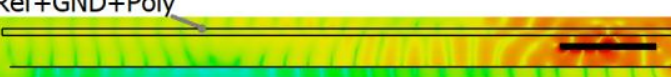

e)

Figura 5 Intensidad de campo eléctrico cercano para: a) Antena acoplada por apertura de referencia. b) Antena de referencia dentro del teléfono mas plano de tierra. c) Antena de referencia en el teléfono mas plano de tierra mas capa protectora de FR4, d)Antena 
de referencia en el teléfono mas plano de tierra mas capa de Teflón, e) Antena de referencia en el teléfono mas plano de tierra mas capa de Policarbonato.

En las Figura 5.a y b, es posible observar que, en el caso de las antenas de referencia con y sin plano de tierra las ondas se propagan con una dirección perpendicular a la antena con intensidades máximas que llegan a los $46658.1 \mathrm{~V} / \mathrm{m}$ (Ref) y $51454.1 \mathrm{~V} / \mathrm{m}$ (Ref+GND) en el plano en mención. Para el caso de las Figura 5c, d y e, las intensidades de campo cercano E se incrementan hasta valores de $56780.9 \mathrm{~V} / \mathrm{m}$ (Poly), $56916.3 \mathrm{~V} / \mathrm{m}$ (PTFE) y $56455,6 \mathrm{~V} / \mathrm{m}$ (FR4), lo cual indica que la estructura del teléfono celular esta desviando la propagación de ondas y la concentra en la estructura, entre la capa protectora y el plano de tierra. Es mas las capas protectoras funcionan como guías de onda, no deseadas, que llevan a la onda hasta los bordes inferior y superior del teléfono. Este efecto se produce también el los otros planos YZ y la razón por la que el patrón de radiación (Figura 4) presenta mayor cantidad de lóbulos laterales y con mayor ganancia. En el presente caso y de manera similar al análisis del patrón de radiación, la capa de Teflón se muestra como la alternativa que menos perjudica a la propagación de ondas, como se ve en la Figura 5.d, donde los frentes de onda dentro de la capa son mas notorios en comparación con los otros dos casos, donde el frente de onda se pierde casi totalmente.

\section{d). Ganancia realizada}

La ganancia realizada es una medida como se distribuye la intensidad de campo eléctrico de manera espacial y los resultados de la simulación se presentan en la Figura 6, para los planos $\phi=0$ y $\phi=90^{\circ}$. La ganancia se representa para tres frecuencias: $24 \mathrm{GHz}, 26$ $\mathrm{GHz}$ y $28 \mathrm{GHz}$. Los lóbulos primarios de las Figura 6.a y ,b son similares para las tres frecuencias, con una máxima de $5.3 \mathrm{~dB}$ en ambos planos. Este valor se utiliza para graficar el circulo de referencia (violeta) para las Figura 6.c, d y e. 

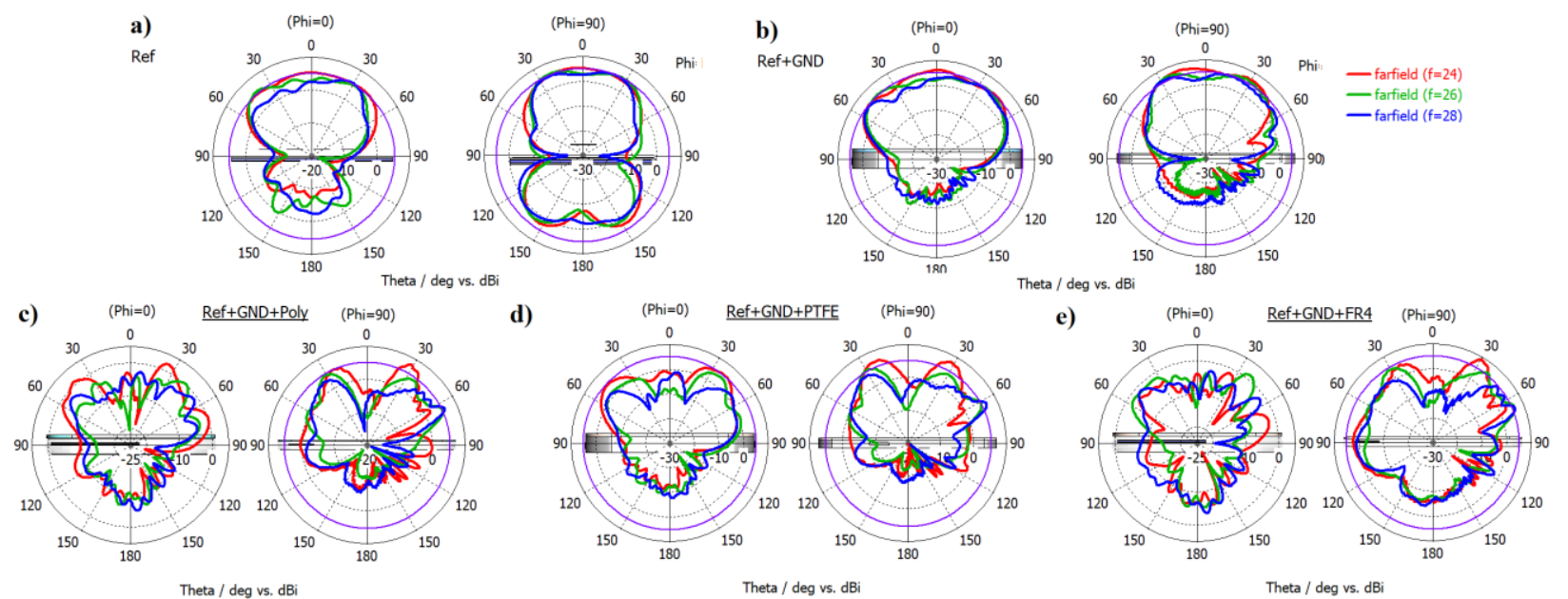

Figura 6 Ganancia realizada para los planos $\boldsymbol{\phi}=\mathbf{0}$ y $\boldsymbol{\phi}=\mathbf{9 0}^{\circ}$ para: a) Antena acoplada por apertura de referencia. b) Antena de referencia dentro del teléfono mas plano de tierra. c) Antena de referencia en el teléfono mas plano de tierra mas capa protectora de Poly, d)Antena de referencia en el teléfono mas plano de tierra mas capa de Teflón, e) Antena de referencia en el teléfono mas plano de tierra mas capa de FR4.

A parte de la confirmación de lo observado en secciones anteriores, podemos mencionar algunas observaciones. En el caso de la Figura 6.d se puede observar, que a pesar de que la orientación del lóbulo principal ha cambiado, la ganancia máxima ha aumentado hasta un valor de $7.93 \mathrm{~dB}$ en la dirección $\theta=28^{\circ}$, en el plano $\phi=90^{\circ}$, para $24 \mathrm{GHz}$. En el plano ortogonal, la ganancia se mantiene cercana al circulo de referencia. En el caso de los resultados para otras capas de FR4 y Poly, también la ganancia máxima en el plano $\phi=90^{\circ}$ llega a valores de $8.85 \mathrm{~dB}$ (FR4) y $7.95 \mathrm{~dB}$ (Poly), para $24 \mathrm{GHz}$. Sin embargo, en el plano ortogonal, los valores de ganancia disminuyen considerablemente en comparación al circulo de referencia. Los resultados confirman que la capas protectoras de menor permitividad, son las que producen efectos menos significativos en la propagación de los campos eléctricos en las distancias cercanas y que luego se traduce en patrones de radiación con múltiples lóbulos. Es necesario subrayar que la capa hecha con Teflón es la que menos modifica la forma del patrón y reduce menos la ganancia máxima, en ambos planos y en todas las frecuencias. Adicionalmente, es posible notar que frecuencias inferiores, tanto la capa de protección de FR4 y de Policarbonato pueden producir haces o lóbulos con alta ganancia.

\section{Conclusiones}


Se realizo la evaluación de los efectos de capas protectoras y el encapsulado estructural de un teléfono, sobre su antena operando en el rango de frecuencias milimétricas. El evaluación de los efectos de dichas capas en el ancho de banda y frecuencia de operación, mostraron variaciones importantes, con reducciones de hasta $83 \%$ con respecto a la banda inicial de la antena de referencia: $4.87 \mathrm{GHz}$. En el caso del patrón de radiación, las capas producen un efecto notorio en la aparición de nodos en la dirección del eje Z, además de producir numerosos lóbulos laterales, siendo la capa de Teflón la que menos perjudica la propagación en la dirección perpendicular a la antena. Analizando las intensidades de campo eléctrico en la estructura, se puede concluir que el teléfono y el plano de tierra se comportan como una guía de onda, debido al tamaño de la longitud de onda. Lo mismo pasa con las capas protectoras, y desvían los frentes de onda hacia los bordes del teléfono, donde la capa de Teflón es la reduce en menor porcentaje dichos frentes de onda en comparación a las otras dos capas. En el caso de la ganancia realizada a lo largo de diferentes frecuencias, se puede verificar que, las capas con permitividad relativa alta, producen haces directivos de hasta $8.85 \mathrm{~dB}$, que pueden ser aprovechados en mejores diseños para protección del teléfono. Por otro lado, una combinación de los tres materiales puede ser una solución interesante, ya que si uno coloca una capa con varios materiales puede mejorar los parámetros espaciales de la antena. Materiales con baja permitividad puede utilizarse en una apertura o ventana ubicada en contacto con los parches y materiales con alta permitividad alrededor de la ventana pueden contribuir a concentrar la intensidad de campo en la dirección Z.

\section{Referencias}

Beltran, E. M. (2019). Desarrollo de antenas miniatura UWB con aplicación en sistemas de comunicaciones móviles $5 \mathrm{G}$.

Elkashlan, M., Duong, T. Q., \& Chen, H. H. (2015). Millimeter-wave communications for 5G-Part 2: applications [Guest Editorial]. IEEE Communications Magazine, 53(1), 166-167.

Farooq U., Rather G.M. (2019) Millimeter Wave (MMW) Communications for Fifth Generation (5G) Mobile Networks. In: Panigrahi C., Pujari A., Misra S., Pati B., Li KC. (eds) Progress in Advanced Computing and Intelligent Engineering. Advances in Intelligent Systems and Computing, vol 714. Springer, Singapore. https://doi.org/10.1007/978-981-13-0224-4_9. 
ITU. (2020). "Status and trends of global 5g spectrum, ". [Online]. Available:URLhttps://www.itu.int/en/ITU-D/Regional-

Presence/AsiaPacific/SiteAssets/Pages//Session\%202-5.pdf

Lak, A., Adelpour, Z., Oraizi, H., \& Parhizgar, N. (2021). Three Configurations of Compact Planar Multistub Microstrip Antennas for mmW Mobile Applications. International Journal of Antennas and Propagation, 2021.

Lin, W., \& Ziolkowski, R. W. (2020). High-Directivity, Compact, Omnidirectional Horizontally Polarized Antenna Array. IEEE Transactions on Antennas and Propagation, 68(8), 6049-6058.

Marcus, M. J. (2015). 5G and" IMT for 2020 and beyond"[Spectrum Policy and Regulatory Issues]. IEEE Wireless Communications, 22(4), 2-3

Nguyen, T. Q. K., Miah, M. S., Lizzi, L., Haneda, K., \& Ferrero, F. (2020). Experimental Evaluation of User's Finger Effects on a 5G Terminal Antenna Array at 26 GHz. IEEE Antennas and Wireless Propagation Letters, 19(6), 892-896.

Niu, Y., Li, Y., Jin, D., Su, L., \& Vasilakos, A. V. (2015). A survey of millimeter wave communications (mmWave) for 5G: opportunities and challenges. Wireless networks, 21(8), 2657-2676.

Pan, H. K., Ruberto, M., Horine, B., \& Ravid, S. (2012). U.S. Patent Application No. $13 / 420,865$.

Rappaport, T. S., Sun, S., Mayzus, R., Zhao, H., Azar, Y., Wang, K., ... \& Gutierrez, F. (2013). Millimeter wave mobile communications for 5G cellular: It will work!. IEEE access, 1, 335-349.

Roh, W., Seol, J. Y., Park, J., Lee, B., Lee, J., Kim, Y., ... \& Aryanfar, F. (2014). Millimeter-wave beamforming as an enabling technology for 5G cellular communications: Theoretical feasibility and prototype results. IEEE communications magazine, 52(2), 106-113.

Sacco, G., Nikolayev, D., Sauleau, R., \& Zhadobov, M. (2021). Antenna/Human Body Coupling in 5G Millimeter-Wave Bands: Do Age and Clothing Matter?. IEEE Journal of Microwaves, 1(2), 593-600. 
Samsung. (2020). "The Vision of 6G". Available online: https://cdn.codeground.org/nsr/downloads/researchareas/20201201_6G_Vision_web.pd $\underline{\mathrm{f}}$ (accessed on 11 May).

Sun, X. (2018). Planar antenna array on LTCC and rogers substrate for 5G applications (Doctoral dissertation, Universidad Politécnica de Madrid).

Zhang, Y. P., \& Liu, D. (2009). Antenna-on-chip and antenna-in-package solutions to highly integrated millimeter-wave devices for wireless communications. IEEE transactions on antennas and propagation, 57(10), 2830-2841. 